\title{
NEVO MELANOCÍTICO CONGÊNITO GIGANTE COM NEUROTIZAÇÃO: RELATO DE CASO
}

\section{ARTIGO ORIGINAL}

ROCHA, Ana Paula Coelho', ZÁU, Aline Sales Mendes², SANTOS, Luciana Mendes $\operatorname{dos}^{3}$

ROCHA, Ana Paula Coelho. ZÁU, Aline Sales Mendes. SANTOS, Luciana Mendes dos. Nevo melanocítico congênito gigante com neurotização: relato de caso. Revista Científica Multidisciplinar Núcleo do Conhecimento. Ano. 07, Ed. 01, Vol. 06, pp. 123-132. Janeiro de 2022. ISSN: 2448-0959, Link de acesso: https://www.nucleodoconhecimento.com.br/saude/congenito-gigante, $\quad$ DOI: 10.32749/nucleodoconhecimento.com.br/saude/congenito-gigante

\section{RESUMO}

Os Nevos Melanocíticos Congênitos (NMC) são hamartomas raros que se caracterizam por proliferações clonais de melanócitos derivados da crista neural, com arranjo em ninhos na epiderme/derme após sua migração durante a embriogênese. Clinicamente são lesões pigmentadas, com limites bem definidos, de tamanho e aspecto variáveis. Denomina-se Nevo Melanocítico Congênito Gigante (NMCG) aquele com vinte ou mais centímetros de diâmetro estimados para a idade adulta. A presença de um NMCG está associada ao maior risco de comorbidades graves como Melanocitose Neurocutânea e Melanoma, devendo-se ainda, excluir o Melanoma Maligno Congênito, principal diagnóstico diferencial. Neste cenário, a presença de nódulos proliferativos seria um fator de pior prognóstico? Este relato tem por objetivo apresentar um caso clínico desafiador de NMCG com nódulos proliferativos, de rápido crescimento, em um recém-nascido com 18 dias de vida. Realizou-se breve revisão

\footnotetext{
${ }^{1}$ Residente do terceiro ano de Dermatologia, Bacharel em Medicina. ORCID: 0000-0001-9895-706X.

${ }^{2}$ Residente do terceiro ano de Dermatologia, Bacharel em Medicina. ORCID: 0000-0002-6561-376X.

${ }^{3}$ Orientadora. Doutora em Doenças Tropicais e Infecciosas pela Universidade do Estado do Amazonas em convênio com a Fundação de Medicina Tropical-FMT-HVD, Especialista em Dermatologia, Bacharel em Medicina. ORCID: 0000-0002-3991-6732.
}

$\mathrm{RC}: 105997$

Disponível em: https://www.nucleodoconhecimento.com.br/saude/congenito-gigante 
de literatura e a compilação de dados clínicos e histopatológicos do paciente em questão, que foi atendido em um ambulatório de Dermatologia. Conclui-se que apesar de existirem características clínicas que apontem para um pior prognóstico ou possível malignização, são fundamentais a interpretação histológica minuciosa e a correta correlação clínico-patológica para que haja diagnóstico e manejo terapêutico adequados.

Palavras-chave: melanoma congênito, nevo congênito gigante, nevo melanocítico, neurotização, nódulos proliferativos.

\section{INTRODUÇÃO}

Os Nevos Melanocíticos Congênitos (NMC) se caracterizam por proliferações benignas clonais de melanócitos, derivados da crista neural, que sofrem alterações na maturação e diferenciação durante a fase de migração no período da embriogênese. As células névicas formam ninhos ou se encontram dispersas na epiderme e derme profunda, em qualquer área do tegumento. Cerca de 1 a $2 \%$ da população exibe estas alterações ao nascimento (SCALVENZI, 2012).

Para diferenciação inicial entre NMC e Nevo Melanocítico Adquirido (NMA), leva-se em consideração o tamanho, a precocidade da lesão e as características histológicas de ambos. Os NMC tendem a ser maiores e estão presentes desde o nascimento ou, em geral, até os primeiros 6 meses de vida (VIANA, 2013). No histopatológico, há controvérsias sobre indicadores reais de diferenciação entre os nevos congênitos e adquiridos, sendo muitas vezes impossível distingui-los. No entanto, ao contrário dos NMA, os NMC não se restringem à junção dermo-epidérmica, apresentam maior celularidade e acometem planos mais profundos (ZAAL, 2004).

De acordo com Simons (2017), na histopatologia dos NMC, os ninhos de melanócitos e as células névicas são encontrados dispersos em diferentes profundidades da derme, entre os feixes de colágeno, ao redor dos anexos, nervos e vasos sanguíneos, podendo infiltrar tecido subcutâneo, músculo e fáscia. Frequentemente há alta celularidade, variados graus de desorganização arquitetural e atipias, bem como 
disseminação pagetoide. Tais achados são comumente encontrados no melanoma, o que aumenta a dificuldade de diferenciação entre ambos e pode levar a equívocos no diagnóstico.

Clinicamente apresentam-se como placas marrom-acastanhadas, fortemente pigmentadas, de limites bem definidos, superfície homogênea e frequentemente mamilonada, com áreas de hipertricose (MADRIGAL DÍEZ, 2015). Contudo, nevos maiores podem ser assimétricos, com bordas irregulares e superfície heterogênea, rugosa ou nodular em virtude do excesso de melanócitos e de neurotização. Este termo se refere ao processo de maturação das células névicas, que ocorre da superfície para a profundidade do tecido, onde se tornam mais fusiformes. Estas se assemelham às células de Schwann que compõem o neurofibroma, tumor pápulonodular de consistência firme, cuja origem embriológica é, também, na crista neural (SINGH, 2015).

Segundo Kopf (1979) os NMC podem ser classificados pelo tamanho, sendo considerados pequenos quando menores que $1,5 \mathrm{~cm}$, médios se entre 1,5 e 19,9 cm e gigantes quando maiores ou iguais a $20 \mathrm{~cm}$, estimados para a idade adulta. Mais recentemente Krengel (2012) propôs uma nova classificação incluindo, além do tamanho, fatores clínicos com a intenção de tornar as descrições mais padronizadas e assertivas quanto ao risco de melanoma. Neste contexto, NMCG que apresentem maior rugosidade, pigmentação mais escura, hipertricose, nódulos subcutâneos e presença de nevos satélites são considerados de pior prognóstico, com risco mais elevado de malignização.

Neste cenário, a presença de nódulos proliferativos seria um fator de pior prognóstico? Este relato tem por objetivo apresentar um caso clínico raro e desafiador de NMCG com nódulos proliferativos, de rápido crescimento, em um recém-nascido com 18 dias de vida, através da exposição de dados clínicos e histológicos do paciente em questão e de breve revisão da literatura com o intuito de esclarecer a relação entre nódulos proliferativos/neurotização e prognóstico. 


\section{RELATO DE CASO}

RN do sexo feminino, 18 dias de vida, natural do Amazonas, nascida a termo, pesando $4.415 \mathrm{~kg}$. Genitora relata cesárea devido a gestação de alto risco, complicada por Préeclâmpsia, Diabetes Gestacional e Infecção do Trato Urinário de repetição, com múltiplos esquemas antibióticos.

Apresenta desde o nascimento placa enegrecida de aspecto aveludado, em hemitórax esquerdo, estendendo-se desde a linha médioclavicular até região dorsal interescapular ipsilateral, medindo $23 \mathrm{~cm}$ no maior diâmetro, com limites bem definidos, presença de pelos em periferia, associada a nodulações centrais e coalescentes, enegrecidas a rosadas variando de $0,5 \mathrm{~cm}$ a $1,5 \mathrm{~cm}$ e consistência amolecida (Figura 1). Há, ainda, lesão satélite, de aproximadamente $0,7 \mathrm{~cm}$, em coxa esquerda (Figura 2). Após 30 dias do atendimento inicial, houve crescimento expressivo das nodulações citadas (Figura 3 ). O restante do exame físico não apresentava alterações e o desenvolvimento neuropsicomotor era adequado para idade. Não havia lesões maternas suspeitas.

Após atendimento dermatológico inicial, optou-se por biópsia incisional de uma lesão nodular central, devido aspecto clínico sugestivo de pior prognóstico. No entanto, o laudo anatomopatológico evidenciou somente características benignas como paraceratose, ninhos de células névicas sobrejacentes a componente dérmico de células névicas ordenadamente dispostas, pigmento melânico até derme profunda e áreas de diferenciação neural, sem atipias. Desta forma, firmou-se o diagnóstico de NMC com neurotização e afastou-se possível neoplasia maligna. Assim, em conjunto com o setor de Cirurgia Pediátrica, optou-se por remoção seriada dos nódulos e seguimento clínico para avaliação periódica do nevo. 
Figura 1. Nevo melanocítico congênito gigante: placa enegrecida, de superfície aveludada, bem delimitada, pilosa, com tumorações centrais, com mais de $20 \mathrm{~cm}$.

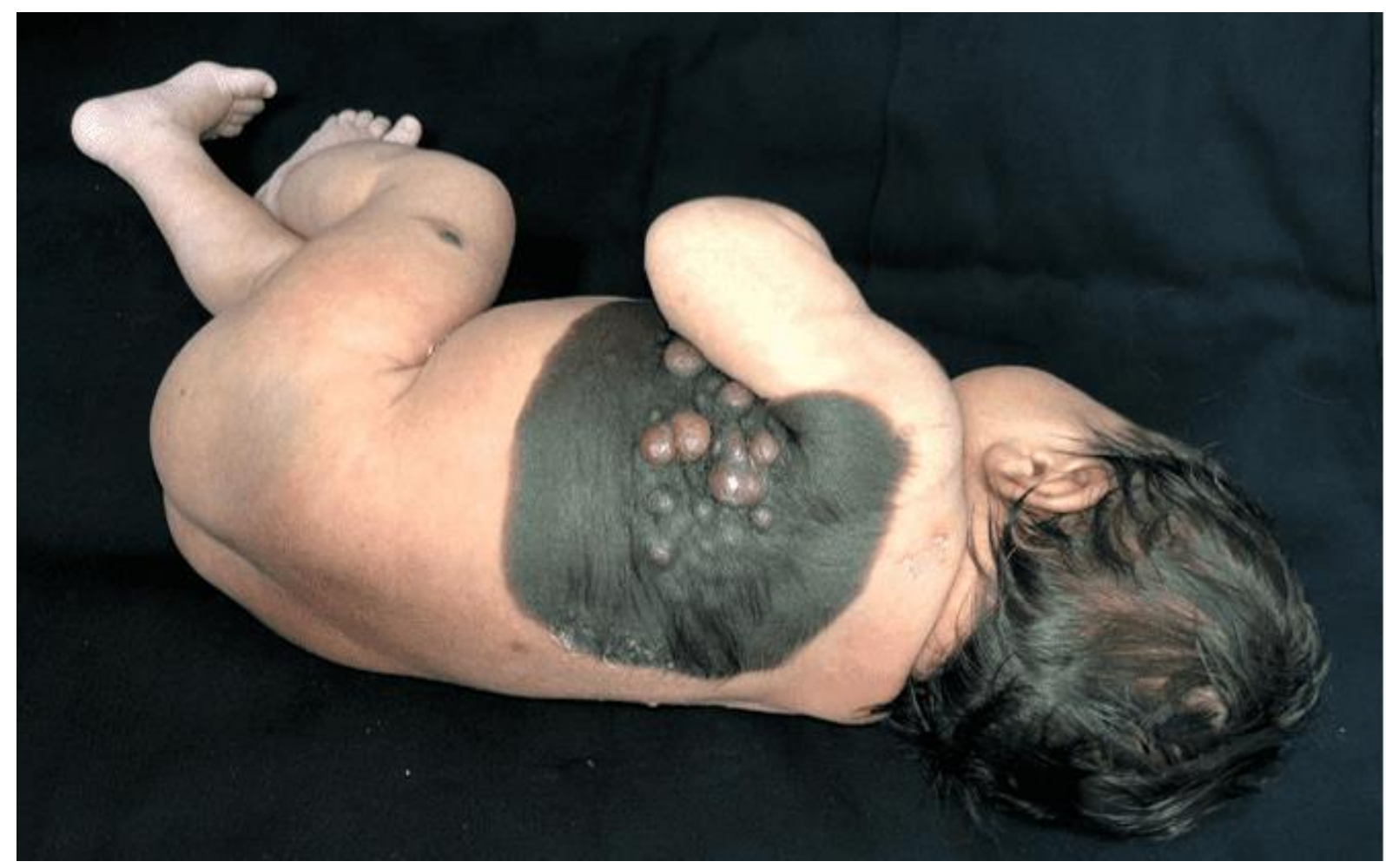

Fonte: arquivo pessoal, 2019. 
Figura 2. Lesão satélite única em coxa esquerda.

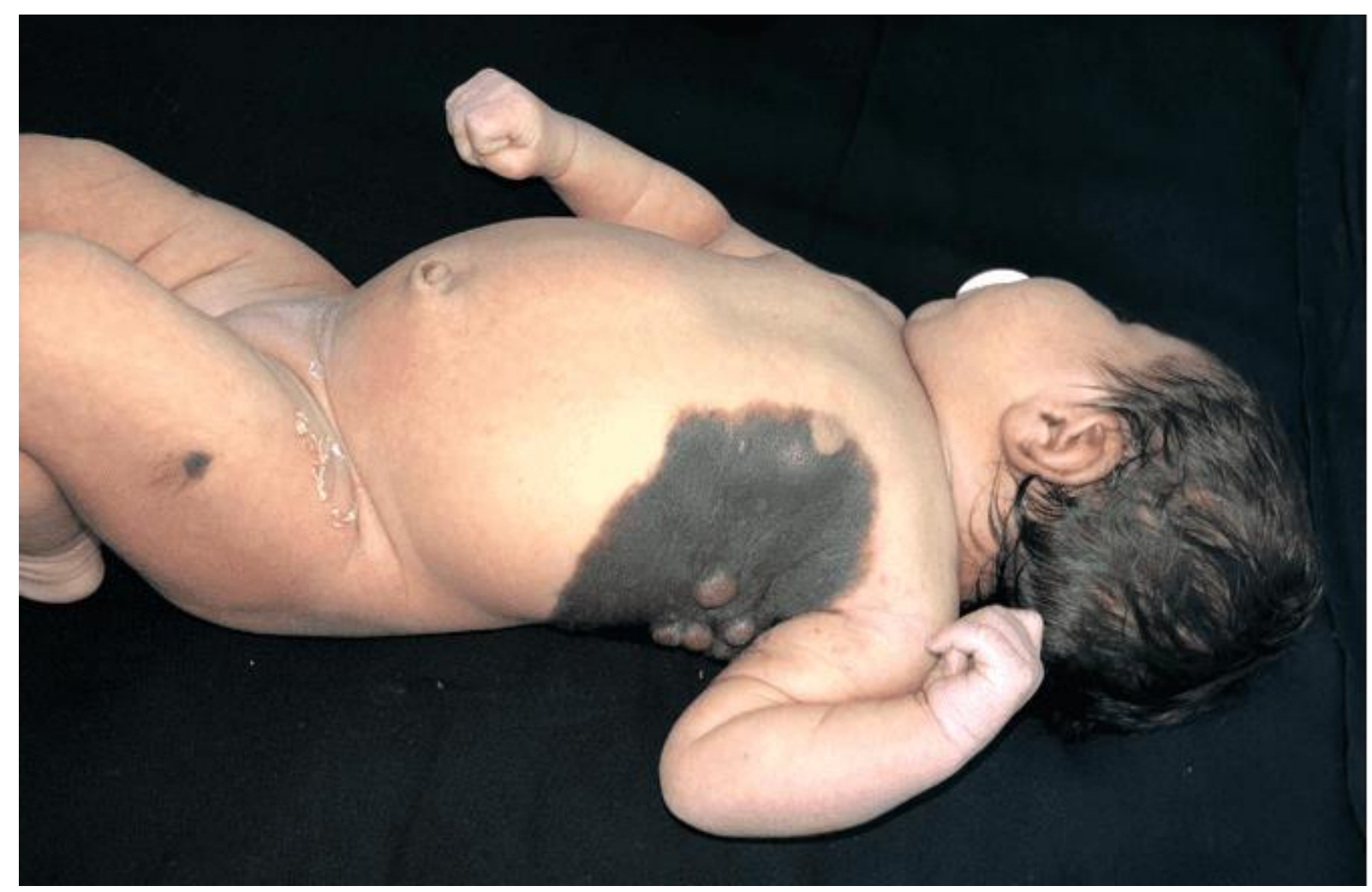

Fonte: arquivo pessoal, 2019.

RC: 105997

Disponível em: https://www.nucleodoconhecimento.com.br/saude/congenito-gigante 
Figura 3. Crescimento rápido das lesões após 30 dias do atendimento inicial, formando tumorações confluentes de consistência amolecida e coloração rosada, mantendo áreas enegrecidas na superfície.

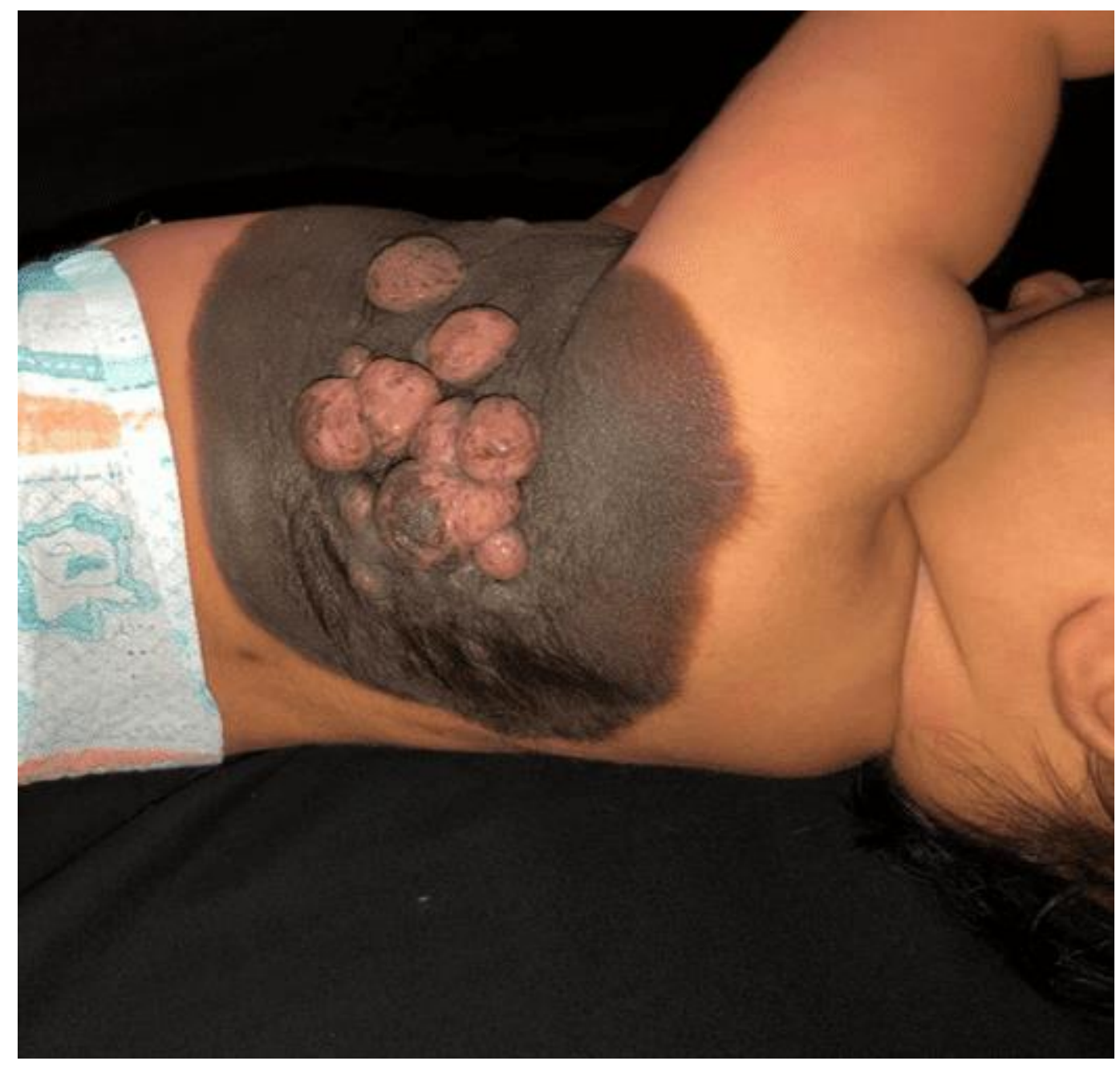

Fonte: arquivo pessoal, 2019.

\section{DISCUSSÃO}

O Nevo Melanocítico Congênito Gigante (NMCG) é uma entidade rara e sua taxa de incidência média é de 1 para cada 20.000 nascidos (ESCADÓN-PÉREZ, 2019). No entanto, apresenta grande importância clínica visto estar associado ao maior risco de comorbidades graves como Melanocitose Neurocutânea (MNC) e Melanoma (RUGGIERI, 2020). A primeira é uma síndrome que acomete o sistema nervoso central e que pode levar a quadros de hipertensão intracraniana e convulsões, 
principalmente quando o NMCG apresenta nodulações, múltiplas lesões satélites e ocorre em localizações como pescoço, couro cabeludo e linha média dorsal. Segundo Alikhan (2012), a taxa de incidência da MNC varia entre 2,5\% e 45\%. Sabe-se que os melanomas surgem primordialmente a partir de melanócitos normais da JDE, no entanto, Zaal (2003) aponta que até $11 \%$ dos NMCG podem evoluir com degeneração maligna, o que ocorre na primeira década de vida em até $60 \%$ dos casos.

A similaridade clínica e dermatoscópica entre o NMCG com nódulos proliferativos e o Melanoma Maligno Congênito ( $M M C)$, entidade rara, mas potencialmente fatal, requer uma interpretação criteriosa dos achados histopatológicos (SCALVENZI, 2012).

Este relato evidencia a presença de um NMCG sem lesões satélites significativas e com marcada presença de nodulações, o que em geral está presente em apenas $3 \%$ dos casos citados na literatura (SCALVENZI, 2012). Os achados anatomopatológicos corroboram o fato de que nódulos dérmicos proliferativos não são, de forma independente, indicativos de malignidade (SIMONS, 2017). Ainda assim, encontra pares no que diz respeito a localização mais comum em tronco, a ausência de história familiar e histopatológico favorável ao seguimento clínico, visto em $75 \%$ dos casos (VIANA, 2017).

Desta forma, há grandes questionamentos acerca da real incidência de Melanoma proveniente de casos de NMCG, bem como da necessidade de intervenção cirúrgica nos casos clinicamente mais desafiadores, seja pelo tamanho da lesão ou pela presença de múltiplos nevos satélites (VIANA, 2017). Ainda não há evidência que comprove o benefício dessa prática como profilaxia ao desenvolvimento de malignidade, além do que, a depender da profundidade das células névicas, não há possibilidade de exérese total e, portanto, cura completa.

Não obstante o desafio terapêutico, o NMCG torna-se, ainda, um desafio diagnóstico pela capacidade de mimetizar clínica e histologicamente o MMC (ESCADÓN-PÉREZ, 2019). 
Ressalta-se que a presença concomitante de múltiplos nevos satélites deve alertar para a possibilidade de acometimento meníngeo e de tumores malignos ou benignos do sistema nervoso central. Neste cenário, faz-se necessária a solicitação de exames complementares, como ressonância magnética ou avaliação do líquor, para melhor avaliação e seguimento do quadro, visto seu pior prognóstico e associação com distúrbios neurológicos potencialmente fatais (RUGGIERI, 2020).

Por fim, o NMCG é responsável por prejuízo estético e social gerando grande impacto emocional aos envolvidos (ZAAL, 2012).

\section{CONSIDERAÇÕES FINAIS}

Diante dos achados na literatura e respondendo a questão norteadora, conclui-se que os nódulos proliferativos/neurotizados são importantes na avaliação clínica do prognóstico dos NMCG, no entanto, não são capazes de determinar um pior desfecho de forma isolada. Os NMCG apresentam os mais variados fenótipos, os quais variam em termos de risco de desenvolvimento de melanoma ou MNC, de dificuldade cirúrgica e de viabilidade do acompanhamento clínico exclusivo. Desta forma, a análise criteriosa do conjunto de informações clínicas combinadas aos achados histopatológicos se faz fundamental.

Tais fatos ilustram a importância de se aprofundar no estudo do NMCG, evento raro, porém, com potencial de acrescentar grande morbidade ao paciente, bem como de impor obstáculos ao diagnóstico e tratamento, que deve primar pela individualidade do caso e ser elaborado de forma multidisciplinar.

\section{REFERÊNCIAS}

ALIKHAN, Ali; IBRAHIMI, Omar A.; EISEN, Daniel B. Congenital melanocytic nevi: where are we now?: part I. Clinical presentation, epidemiology, pathogenesis, histology, malignant transformation, and neurocutaneous melanosis. Journal of the American Academy of Dermatology, v. 67, n. 4, p. 495. e1-495. e17, 2012. 
COTTON, Colleen H.; GOLDBERG, Gerald N. Evolution of congenital melanocytic nevi toward benignity: a case series. Pediatric dermatology, v. 36, n. 2, p. 227-231, 2019.

ESCANDÓN-PÉREZ, Sabrina et al. Nevo melanocítico congénito gigante. Boletín médico del Hospital Infantil de México, v. 76, n. 6, p. 251-258, 2019.

KRENGEL, Sven et al. New recommendations for the categorization of cutaneous features of congenital melanocytic nevi. Journal of the American Academy of Dermatology, v. 68, n. 3, p. 441-451, 2013.

KOPF, Alfred W.; BART, Robert S.; HENNESSEY, Patrick. Congenital nevocytic nevi and malignant melanomas. Journal of the American Academy of Dermatology, v. 1, n. 2, p. 123-130, 1979.

MADRIGAL DÍEZ, C. et al. Nevo melanocítico congénito gigante. Pediatría Atención Primaria, v. 17, n. 68, p. 351-355, 2015.

RUGGIERI, Martino et al. Neurocutaneous melanocytosis (melanosis). Child's Nervous System, v. 36, n. 10, p. 2571-2596, 2020.

SCALVENZI, Massimiliano et al. Giant congenital melanocytic naevus with proliferative nodules mimicking congenital malignant melanoma: a case report and review of the literature of congenital melanoma. Case reports in dermatological medicine, v. 2013, 2013.

SIMONS, Emily A.; HUANG, Jennifer T.; SCHMIDT, Birgitta. Congenital melanocytic nevi in young children: Histopathologic features and clinical outcomes. Journal of the American Academy of Dermatology, v. 76, n. 5, p. 941-947, 2017.

SINGH, Nidhi et al. Neurotized congenital melanocytic nevus resembling a pigmented neurofibroma. Indian journal of dermatology, v. 60, n. 1, p. 46, 2015. 
VIANA, Ana Carolina Leite; GONTIJO, Bernardo; BITTENCOURT, Flávia Vasques. Giant congenital melanocytic nevus. Anais brasileiros de dermatologia, v. 88, p. 863-878, 2013.

VIANA, Ana Carolina Leite et al. A prospective study of patients with large congenital melanocytic nevi and the risk of melanoma. Anais brasileiros de dermatologia, $v$. 92, p. 200-205, 2017.

ZAAL, L. H. et al. Classification of congenital melanocytic naevi and malignant transformation: a review of the literature. British journal of plastic surgery, v. $57, \mathrm{n}$. 8, p. 707-719, 2004.

Enviado: Janeiro, 2022.

Aprovado: Janeiro, 2022. 